\title{
Otimização sob incerteza de sistemas de produção: Interação lavoura-pecuária, com enfase em bovinocultura de leite
}

\author{
Optimization under uncertainty of farm systems: interaction between crop and animal \\ production, with emphasis in dairy cattle
}

\section{Benedito Silva Neto $^{1}$ Eliani Retzlaff ${ }^{2}$}

\section{RESUMO}

$\mathrm{Na}$ modelagem de sistema de produção agropecuária, a possibilidade de combinar livremente as pastagens para a composição do sistema de alimentação dificulta a avaliação em termos probabilísticos da bovinocultura de leite. Neste trabalho, propõe-se um modelo de otimização que, utilizando uma abordagem não probabilista da incerteza, permite contornar este problema. A aplicação do modelo para a análise de uma unidade de produção agropecuária indicou que uma diminuição das culturas de grãos comerciais com a constituição de reservas de pastagens pode se constituir em uma estratégia interessante para minimizar as perdas econômicas em caso de queda de rendimento físico das forrageiras. Além disto, pelo fato de dispensar pressupostos estatísticos e basear-se em cenários que representam condições técnicas e econômicas que puderam ser facilmente discutidas com o agricultor, concluiu-se que a abordagem utilizada neste trabalho pode se constituir em um procedimento útil no planejamento de sistemas de produção sob incerteza.

Palavras-chave: modelagem da incerteza, sistemas de produção agropecuária, bovinocultura de leite, programação matemática.

\section{ABSTRACT}

In the farm systems modeling, the possibility of combining the pastures freely for the composition of the feeding system hinders the probabilistic evaluation of the dairy production. In this work an optimization model with a nonprobabilistic approach of the uncertainty is proposed that permits to handle this problem. The application of the model for the analysis of a unit of agricultural production indicated that a decrease of the cultures of commercial grains with the constitution of pastures reserve could be an interesting strategy to minimize the economic losses if the physical revenue of the forage diminishes. Besides, because the non-statistical uncertainty modeling and the formulation of scenarios that represent technical and economic conditions that could be discussed easily with the farmer, the approach used in this work can be an useful procedure in the planning of farm systems under uncertainty.

Key words: uncertainty modeling, farm systems, dairy production, mathematical programming.

\section{INTRODUÇÃO}

A otimização de sistema de produção agropecuária é usualmente efetuada através de modelos probabilísticos como o modelo de minimização da variância proposto por MARKOWITZ (1952) e o modelo de minimização do desvio médio absoluto de HAZELL, (1971). Tais modelos exigem a disponibilidade de séries históricas dos resultados econômicos ou, pelo menos, estimativas adequadas da medida estatística dos desvios das variáveis relacionadas ao resultado econômico (variância no modelo de MARKOWITZ (1952) e desvio absoluto no modelo de HAZELL (1971)). Assim, nestes modelos, as variações dos rendimentos das culturas e das pastagens têm que ser definidas em termos econômicos e não apenas físicos.

Entretanto, em sistemas que envolvem a bovinocultura de leite, especialmente quando esta é realizada a pasto e se deseja manter um alto grau de liberdade para a composição do sistema de alimentação, as conseqüências econômicas das variações dos rendimentos físicos de cada pastagem

${ }^{1}$ Engenheiro Agrônomo, Doutor em Desenvolvimento Agrícola, professor do Departamento de Estudos Agrários da Universidade Regional do Noroeste do EStado do Rio Grande do Sul (UNÍJUI), 98700-000, Ijuí, RS. E-mail: bsneto@ uniji.tche.br. Autor para correspondência. ${ }^{2}$ Bacharel em Matemática, Mestre em Modelagem Matemática. 
não podem ser estimadas antes do processo de otimização. Isto porque o papel de cada pastagem no fornecimento de alimento ao rebanho depende das demais pastagens presentes no sistema de alimentação. Por exemplo, se uma pastagem é responsável por todo o fornecimento de alimento ao rebanho, durante todo o ano, ela desempenhará um papel diferente do caso em que, a partir da sua combinação com outra fonte de alimento, ela for responsável pelo fornecimento de alimento apenas em um período do ano. Assim, uma mesma queda de rendimento desta pastagem terá uma repercussão diferente, em cada um destes casos, sobre a produção de leite. Teoricamente, do ponto de vista de um agente econômico que toma suas decisões baseado em uma abordagem probabilista, esta situação pode ser caracterizada como de "incerteza processual" (DOSI \& EGIDI, 1992), ou seja, o agente não é capaz de chegar a solução ótima do problema devido à impossibilidade de um processamento adequado das informações.

Neste trabalho, procurou-se desenvolver um modelo que, contornando o problema exposto acima, permite a otimização de sistemas de produção com bovinocultura de leite a pasto. Neste modelo, as perdas possíveis dos rendimentos das forrageiras são expressas em termos físicos, sendo os seus resultados expressos em relação a situações concretas (cenários) e não em probabilidades, o que deve, inclusive, facilitar a sua compreensão por técnicos e agricultores.

\section{MATERIAL E MÉTODOS}

O desenvolvimento do modelo foi baseado em características comumente encontradas nas unidades de produção da região Noroeste do Rio Grande do Sul que produzem leite. Nestas unidades, as opções de produção compreendem as culturas de soja, milho e trigo (plantio direto e convencional), além do leite, o qual algumas vezes é associado com a bovinocultura de corte. Para a produção de leite, as opções para alimentação do gado são pastagens permanentes, pastagens temporárias de verão e de inverno, silagem, grãos e concentrados.

O critério para otimização adotado no modelo é o maximin, isto é, procura-se obter a solução que propicia o maior resultado econômico na(s) pior(es) das situações previstas (ANDRADE, 2000). Tais situações são descritas através da elaboração de cenários onde são computadas as possíveis perdas que as produções podem sofrer. Uma atenção especial foi dispensada para a consideração das possíveis relações entre as perdas de diferentes atividades (KAHNEMAN \& TVERSKY, 1982a).
Todos os resultados econômicos foram convertidos para o dólar americano, baseando-se na cotação média do real, em dólar comercial para venda (a qual era de R\$1,15/US\$ em 1998, época do levantamento dos dados).

\section{Estrutura formal do modelo}

O modelo desenvolvido pode ser descrito como:

Maximizar $R_{M}$

Sujeito a

A $x<=0$

$B x<=b$

$C x>=R_{M}$

$c x=r$

Onde:

$R_{M}=$ margem bruta anual nos piores cenários;

$x=$ atividades do sistema de produção (animais, pastagens, culturas, concentrados, etc.);

$A=$ matriz envolvendo as restrições de alimentação dos bovinos;

$B=$ matriz das restrições de superfície e mão de obra; $b=$ vetor coluna de restrições relativas à mão de obra e a superfície;

$C=$ matriz de cenários com perdas.

$\bar{c} x=$ vetor linha da margem bruta em condições normais (sem perda);

$r=$ margem bruta obtida em condições normais;

A partir das matrizes A, B, e C, temos os seguintes conjuntos de restrições:

A $x<=0$ (restrições para alimentação dos bovinos). Bovinos de leite:

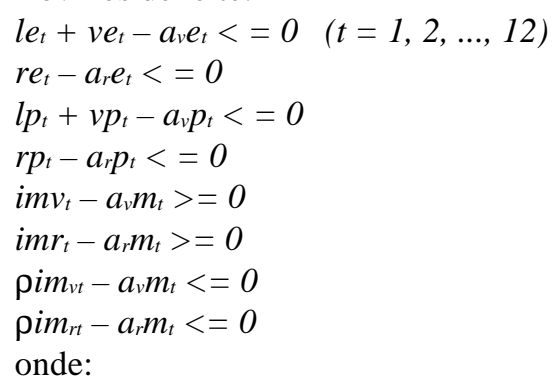

$l e_{t}=$ energia necessária para a produção do leite no mês t;

$v e_{t}=$ energia necessária para manutenção das vacas em lactação no mês t;

$a_{v} e_{t}=$ energia disponível nos alimentos para as vacas em lactação no mês t;

$r e_{t}=$ energia necessária para manutenção dos animais não produtivos no mês t;

$a_{r} e_{t}=$ energia disponível nos alimentos para os animais não produtivos no mês t;

$l p_{t}=$ proteína necessária para produção do leite no mês t; 
$v p_{t}=$ proteína necessária para manutenção das vacas em lactação no mês t;

$a_{v} p_{t}=$ proteína disponível nos alimentos para as vacas em lactação no mês t;

$r p_{t}=$ proteína necessária para manutenção dos animais não produtivos no mês t;

$a_{r} p_{t}=$ proteína disponível nos alimentos para os animais não produtivos no mês t;

$i m v_{t}=$ capacidade de ingestão de matéria seca pelas vacas em lactação no mês t;

$a_{v} m_{t}=$ matéria seca contida nos alimentos para as vacas em lactação no mês t;

$i m r_{t}=$ capacidade de ingestão de matéria seca dos animais não produtivos no mês t;

$a_{r} m_{t}=$ matéria seca contida nos alimentos para os animais não produtivos no mês t;

$i m_{v t}=$ ingestão de volumosos pelas vacas em lactação no mês $\mathrm{t}$;

$a_{v} m_{t}=$ matéria seca contida nos alimentos volumosos para as vacas em lactação no mês t;

$i m_{r t}=$ ingestão de volumosos dos animais não produtivos no mês t;

$a_{r} m_{t}=$ matéria seca contida nos alimentos volumosos dos animais não produtivos no mês t;

$\rho=$ proporção mínima de volumosos na dieta (que é igual a 50\% da capacidade de ingestão).

As restrições de alimentação para o gado de corte também fazem parte deste conjunto:

$c e_{t}-a_{c} e_{t}<=0 \quad(t=1,2, \ldots, 12)$

$c p_{t}-a_{c} p_{t}<=0$

$\operatorname{\rho im}_{c t}-a_{c} m_{t}>=0$

onde:

$c e_{t}=$ energia necessária para manutenção do gado de corte no mês t;

$a_{c} e_{t}=$ energia disponível nos alimentos para o gado de corte no mês t;

$c p_{t}=$ proteína necessária para manutenção do gado de corte no mês t;

$a_{c} p_{t}=$ proteína disponível nos alimentos para o gado de corte no mês t;

$\rho=$ proporção mínima de volumosos na dieta (que é igual a $50 \%$ da capacidade de ingestão).

$\operatorname{im}_{c t}=$ ingestão de volumosos pelo gado de corte no mês t;

$a_{c} m_{t}=$ matéria seca contida nos alimentos volumosos para o gado de corte no mês t;

$\mathrm{B} \mathrm{x}<=\mathrm{b}$, restrições de superfície e de mão de obra, as únicas limitadas por um $\mathrm{b}>0$.

$\mathrm{Cx}>=\mathrm{R}_{\mathrm{M}}$, restrições relativas aos cenários de perdas.

$\mathrm{C} \mathrm{x}$ corresponde aos diferentes cenários, os quais são definidos por:

$\bar{c} \mathrm{x}-P P P L-P P P C>=R_{M}$ (cenário queda do preço do leite e da carne) $\bar{c} \mathrm{x}-\mathrm{PPS}-P P P M>=R_{M}$ (cenário queda do preço da soja e do milho)

$\bar{c} \mathbf{x}-E L P E C A-P P G>=R_{M}$ (cenários estiagem no verão e excesso de chuva no inverno)

cujos termos são definidos como

$E L=(N E L+N E V L) / N T E$

$P E C A=P E C A_{s}+P E C A_{p}$

$P E C A_{s}=\left(\begin{array}{lll}S P P & E M S\end{array}\right) / \mathrm{a} \mathrm{r}$

$P E C A_{p}=(P E M F) / \mathrm{a} \mathrm{r}$

$P=F C-F T \quad P P_{p}$

Onde:

$\bar{c} x=$ vetor da margem bruta em situações normais (sem perda);

$P P P L=$ perda possível devido à queda do preço do leite;

$P P P C=$ perda possível devido à queda do preço da carne;

$P P P S=$ perda possível devido à queda do preço da soja;

$P P P M=$ perda possível devido à queda do preço do milho;

$E L=$ fator de perda de leite em relação às perdas das pastagens

NEL = necessidade de energia para a produção de leite

$\mathrm{NEVL}=$ necessidade de energia para manutenção das vacas em lactação

NTE = necessidade de energia para todo o rebanho leiteiro.

$P E C A$ = perda econômica possível na alimentação dos bovinos;

$P P C=$ perda possível nas culturas de grãos comerciais $P E C A_{s}=$ perda econômica para silagem;

$P E C A_{p}=$ perda econômica das pastagens (verão ou inverno);

$S=$ matéria seca total de silagem;

$E M S=$ teor de energia metabolizável da silagem;

$E M F$ = teor de energia metabolizável da pastagem;

$\mathrm{a}=$ necessidade de energia para produção de um litro de leite;

$\mathrm{r}=$ preço do leite;

$P=$ perda de matéria seca efetiva da pastagem (a que resulta em perdas em leite);

$F C$ = matéria seca consumida da pastagem;

$F T=$ matéria seca total da pastagem;

$P P_{s}=$ perda possível de matéria seca da silagem;

$P P_{p}=$ perda possível de matéria seca em relação ao rendimento físico total da pastagem.

O modelo prevê ainda restrições relativas à formação da quota de leite, rotações de culturas e ligação entre restrições, as quais devem ser formuladas de acordo com as especificidades da unidade de produção e, no 
caso do sistema de quotas, da agroindústria compradora do leite.

\section{Procedimento adotado para a aplicação do modelo em uma unidade de produção}

Este modelo foi utilizado para analisar uma unidade de produção agropecuária localizada no município de Vitória das Missões, em 1.998. Esta unidade de produção dispõe de 150 hectares de superfície agrícola útil e uma mão de obra correspondente a duas unidades de trabalho (agricultor em tempo integral e esposa e filhos em tempo parcial). A partir de entrevistas com o agricultor, foram coletados os dados técnicos (como a quantidade de trabalho demandada pelas atividades, os esquemas de rotação adotados, o sistema de quotas da indústria, a quantidade de insumos utilizada em cada atividade, os rendimentos físicos obtidos, etc.) e econômicos (preços de insumos e produtos). Na literatura especializada, foram coletadas informações sobre os teores de energia e proteína dos alimentos para o gado, disponíveis na unidade de produção (FREITAS e outros, 1994), a necessidade de energia e proteína para cada categoria de animal (NRC, 1989). Quanto à capacidade de ingestão de alimentos dos animais, a qual foi fixada em 3\% do peso vivo, não foi possível, neste trabalho, torná-la flexível. No entanto, como neste modelo considera-se um rebanho em equilíbrio reprodutivo e com partos distribuídos de forma homogênea durante o ano (sendo, por estas razões, o número de animais por categoria mantido constante ao longo do ano), entendemos que a consideração de uma capacidade de ingestão "média" pode se constituir em uma boa aproximação. Além disto, constatamos que a fixação da capacidade de ingestão em $3 \%$ do peso vivo evita que se obtenha rendimentos de leite por vaca e por dia muito elevados, os quais poderiam estar além do potencial genético dos animais disponíveis na propriedade. A partir de entrevistas com o agricultor, foram definidos os seguintes cenários que caracterizam as possíveis situações de perda econômica para a unidade de produção: estiagem no verão, excesso de chuva no inverno e queda do preço do leite e da carne, a qual foi fixada em $20 \%$. Foi também incorporado ao modelo um cenário relativo à queda do preço da soja e do milho, a qual foi fixada em 25\%. Optou-se por considerar nos cenários apenas perdas moderadas de modo que estes pudessem representar situações relativamente freqüentes. A partir destes dados e informações, foi elaborado o modelo completo (com incerteza), o qual é composto de 289 expressões (função objetivo e restrições) e 328 variáveis, e obtida a sua solução. Após, uma nova simulação foi efetuada substituindo-se, na função objetivo, a margem bruta mínima $\left(R_{M}\right)$ pela margem bruta obtida em condições normais $(r)$ o que nos permitiu obter a solução do modelo determinista (sem considerar a incerteza) com o cálculo das perdas possíveis a que o agricultor estaria sujeito com a adoção desta solução. Foi também realizada uma simulação impondo-se uma solução ao modelo completo equivalente ao sistema de produção atualmente praticado pelo agricultor para que se pudesse estimar as perdas as quais ele está sujeito segundo os cenários definidos. Enfim, é interessante observar que, após a definição da estrutura formal do modelo, todo o processo de modelagem da unidade de produção foi concluído em cerca de 2 meses, tendo sido realizado um total de 5 visitas ao agricultor. A modelagem e as simulações foram realizadas no sofware LINGO da LINDO Systems Inc.

\section{RESULTADOS E DISCUSSÃO}

Na tabela 1, encontra-se representada a solução obtida a partir do modelo completo, comparada com a solução do modelo determinista e com os resultados obtidos a partir do sistema de produção atualmente praticado pelo agricultor. Os resultados apresentados na tabela 1 indicam que, caso o agricultor altere o nível das atividades segundo as soluções dos modelos, ele poderia obter um aumento da sua margem bruta de U\$ 9.618, segundo o modelo determinista. Seguindo a solução obtida com o modelo completo o agricultor teria um pequeno aumento, de apenas U\$ 1.120. Por outro lado, adotando a solução do modelo determinista, a margem bruta mínima obtida pelo agricultor seria de U\$ 17.963 , enquanto que, adotando a solução indicada pelo modelo completo, a margem bruta mínima seria de U\$28.022. É interessante notar que, segundo o modelo completo, o agricultor obteria a margem mínima em três cenários de perdas, enquanto que segundo o modelo determinista ele obteria a margem mínima apenas no cenário de estiagem no verão.

Além disto, nos cenários de excesso de chuva no inverno, queda dos preços do leite e da carne e queda dos preços da soja e do milho, adotando a solução do modelo determinista, o agricultor obteria uma margem bruta superior em relação a que ele obteria nestes cenários adotando a solução do modelo completo. Isto faz com que a obtenção de uma margem bruta equivalente à mínima obtida com o modelo completo seja mais provável com a adoção da solução deste modelo do que com a adoção da solução do modelo determinista. Entretanto, considerando-se 
Tabela 1 - Margem bruta anual obtida com o modelo completo, com o sistema de produção atual e com o modelo determinista.

\begin{tabular}{|c|c|c|c|c|}
\hline \multirow{2}{*}{ Descrição } & \multirow{2}{*}{ Unidade de medida } & \multicolumn{3}{|c|}{ Solução } \\
\hline & & Modelo completo & Sistema atual & Modelo determinista \\
\hline Margem bruta em condições normais & dólares $*$ & $37.261,46$ & $36.140,87$ & $45.759,17$ \\
\hline Margem bruta no cenário de estiagem no verão & dólares & $28.021,35$ & $18.343,23$ & $17.962,94$ \\
\hline $\begin{array}{l}\text { Margem bruta no cenário de excesso de chuva no } \\
\text { inverno }\end{array}$ & dólares & $28.021,35$ & $33.385,04$ & $38.303,10$ \\
\hline $\begin{array}{l}\text { Margem bruta no cenário de queda de preço do } \\
\text { leite }\end{array}$ & dólares & $28.021,35$ & $32.826,78$ & $38.171,33$ \\
\hline $\begin{array}{l}\text { Margem bruta no cenário de queda de preço da } \\
\text { soja e do milho }\end{array}$ & dólares & $34.979,08$ & $29.191,97$ & $39.065,16$ \\
\hline Margem bruta mínima & dólares & $28.021,35$ & $18.343,23$ & $17.962,94$ \\
\hline Perda possível & dólares & $9.240,11$ & $17.797,63$ & $27.796,22$ \\
\hline
\end{tabular}

*Observação: cotação baseada no dólar americano comercial para venda (R\$1,15/US\$ em 1998).

Fonte: dados da pesquisa.

apenas as condições normais de produção e o cenário de estiagem no verão, ao adotar a solução do modelo completo, em detrimento da adoção da solução do modelo determinista, o agricultor estaria renunciando à U\$ 8.497 de aumento da sua margem bruta nas condições normais de produção, mas estaria assegurando cerca de U\$ 10.058 a mais caso ocorresse uma estiagem no verão. Assim a adoção da solução do modelo completo pode ser interessante, caso o agricultor esteja em uma situação financeira vulnerável. Por esta razão, considera-se o modelo completo como o mais adequado para a discussão de estratégias para o aumento do resultado econômico da unidade de produção.

A tabela 2 apresenta os níveis das atividades segundo as soluções obtidas nos modelos e na situação atual do agricultor. Pode-se observar que a adoção da solução de qualquer um dos modelos implicaria mudanças drásticas no sistema de produção atual, destacando-se dentre estas o significativo aumento do rendimento das vacas leiteiras proposta na solução de ambos os modelos e a grande expansão da área de pastagem permanente proposta pela solução do modelo completo. Além disto, nos modelos elaborados persistem várias fontes "internas" de incerteza (KANEHMAN \& TVERSKY, 1982b), ou seja, originadas da falta de informações adequadas para a estimativa de certos coeficientes, como, por exemplo, em relação à capacidade de ingestão dos animais e a expressão que define a perda de leite em relação à queda de rendimento das pastagens (fator EL). Assim, uma interpretação em termos estratégicos dos resultados nos modelos talvez seja mais interessante do que a consideração da adoção das soluções, literalmente como elas se apresentam. Neste sentido, é interessante observar que o aumento significativo da margem bruta mínima obtida no modelo completo em relação ao modelo determinista deve-se a uma diminuição das culturas comerciais (de 121,4 ha para no modelo determinista para 41,8 ha no modelo completo), as quais são substituídas por pastagens e silagem. Esta substituição permite a produção de uma reserva de alimentos capaz de compensar eventuais quedas nos rendimentos das pastagens e da silagem, apesar de representar um excedente em condições normais de produção. Ao questionarmos o agricultor, este concordou com a validade de tal estratégia para diminuir a incerteza embora considerasse excessivo o aumento da área de pastagem permanente indicado pela solução do modelo completo.

\section{CONCLUSÕES}

Os resultados obtidos neste trabalho permitem chegar às seguintes conclusões:

A adoção de uma abordagem não probabilista baseada no critério maximin e na construção de cenários permitiu a modelagem da incerteza em sistemas de produção com bovinocultura de leite a pasto.

A diminuição da incerteza na aplicação do modelo a uma unidade de produção com bovinocultura de leite ocorreu através de dois mecanismos, a saber, a substituição das culturas de grãos comerciais (soja e milho) pela atividade leiteira e a constituição de reservas de alimentos, especialmente pastagens, para o gado de leite.

Além disto, pelo fato de dispensar pressupostos estatísticos e basear-se em cenários que representam condições técnicas e econômicas, as estratégias para a limitação das perdas devido à incerteza puderam ser facilmente discutidas com o

Ciência Rural, v.34, n.4, jul-ago, 2004. 
Tabela 2 - Solução do modelo completo comparada com o sistema de produção atual e com a solução do modelo determinista.

\begin{tabular}{|c|c|c|c|c|}
\hline \multirow{2}{*}{ Atividade } & \multirow{2}{*}{ Unidade de medida } & \multicolumn{3}{|c|}{ Solução } \\
\hline & & Modelo completo & Sistema atual & Modelo determinista \\
\hline Leite dentro da quota & litros & $243.922,2$ & 98.280 & 200.719 \\
\hline Leite extraquota & litros & 203,8 & 0 & 43,15 \\
\hline Rendimento de leite por dia por vaca & litros/vaca/dia & 19,7 & 13 & 20,34 \\
\hline Total de bovinos de leite & cabeças & 98 & 50 & 79 \\
\hline Vacas de descarte & cabeças & 14 & 12 & 11,6 \\
\hline Vacas em lactação & cabeças & 34 & 21 & 27,4 \\
\hline Pastagem permanente & hectares & 83,7 & 36 & 6 \\
\hline Aveia cobertura $\mathrm{p} /$ soja & hectares & 14,4 & 32 & 51,8 \\
\hline Aveia cobertura $\mathrm{p} /$ milho & hectares & 14,4 & 8 & 51,8 \\
\hline Aveia + azevém & hectares & 31,5 & 42 & 23,5 \\
\hline Silagem & hectares & 4,2 & 0 & 7 \\
\hline Milho cortado para o gado & hectares & 1,04 & 0 & 0,15 \\
\hline Milheto & hectares & 10,4 & 2 & 6,5 \\
\hline Sorgo & hectares & 7,8 & 9 & 8,1 \\
\hline Resíduo de soja & toneladas & 51,9 & 12,8 & 52,22 \\
\hline Farelo de trigo & toneladas & 0 & 0 & 0 \\
\hline Subsistência & hectares & 1 & 1 & 1 \\
\hline Milho convencional & hectares & 7,8 & 9 & 15,5 \\
\hline Milho plantio direto & hectares & 14,4 & 8 & 51,8 \\
\hline Soja convencional & hectares & 5,2 & 42 & 2,3 \\
\hline Soja plantio direto & hectares & 14,4 & 32 & 51,8 \\
\hline Bovino de corte vendidos & cabeças & 0 & 0 & 0 \\
\hline Vacas de corte & cabeças & 0 & 5 & 0 \\
\hline
\end{tabular}

Fonte: Dados da pesquisa.

agricultor Assim, de maneira geral pode-se concluir que o procedimento para a modelagem sob incerteza proposto neste trabalho pode se constituir em um instrumento útil para o planejamento de sistema de produção agropecuária.

\section{REFERÊNCIAS BIBLIOGRÁFICAS}

ANDRADE, L.E. de Introdução à pesquisa operacional: métodos e modelos para a análise de decisão. Rio de Janeiro : LTC, 2000. 277p.

DOSI, G.; EGIDI, M. Substantive and procedural uncertainty: An exploration of economic behaviours in changing environments. Evolutionary Economics, v.1, p.145-168, 1991.

FREITAS, E.A.G. de; DUFLOTH, J.H.; GREINER, L.C. Tabela de composição químico-bromatológica e energética dos alimentos para animais ruminantes em Santa Catarina. Florianópolis : EPAGRI, 1994. 333p.
HAZELL, P.B.R. A linear alternative to quadratic and semivariance programming for farm planning under uncertainty. American Journal of Agricultural Economics, v.53, n.1, p.5362, 1971.

KAHNEMAN, D.; TVERSKY, A. The simulation heuristic. In: KAHNEMAN, D.; TVERSKY, A.; SLOVIC P. Judgament under uncertainty: heuristic and biases. Cambridge : Cambridge University, 1982a. p.201-208.

KAHNEMAN, D.; TVERSKY, A. Variants of uncertainty. In KAHNEMAN, D.; TVERSKY, A.; SLOVIC P. Judgment under uncertainty: heuristic and biases. Cambridge University, 1982b. p. $510-520$.

MARKOWITZ, H.M. Portfolio selection, Journal of Finance, v.7, p.77-91, 1952.

NATIONAL RESEARCH COUNCIL (NRC): Nutrient requirements of dairy cattle. Washington, DC : National Academy of Sciences, 1989. 253p. 